\title{
Atividade de água, pH, umidade e desenvolvimento de Staphylococcus xylosus durante o processamento e armazenamento da paleta suína curada, maturada e fermentada \\ Water activity, $\mathrm{pH}$, moisture and growth of Staphylococcus xylosus during processing and storage of cured, matured and fermented pork shoulder
}

\author{
Nelcindo Nascimento TERRA ${ }^{1}$, Renato João Sossela de FREITAS² , Alexandre José CICHOSKI ${ }^{3 *}$
}

\begin{abstract}
Resumo
Dez peças de paletas suínas sem pele e sem osso, pesando em média $1,6 \mathrm{~kg}$, receberam injeção de salmoura $\left(20 \%\right.$ p. $\left.\mathrm{v}^{-1}\right)$ contendo cultura de Staphylococcus xylosus e permaneceram dentro de uma câmara durante 30 dias à temperatura de 4 a $9{ }^{\circ} \mathrm{C}$ e umidade de 55 a $45 \%$ controladas Em seguida, foram embaladas a vácuo e armazenadas durante 120 dias a $10^{\circ} \mathrm{C}$. Desde o início da produção até o final do armazenamento, as amostras foram coletadas da superfície e da parte interna das peças de paleta suína, em triplicata, para realização das análises de atividade de água, pH, umidade e contagem da cultura de S. xylosus, com objetivo de caracterizá-las e verificar as possíveis associações com ações exercidas em outros produtos cárneos fermentados. Durante a etapa de armazenamento, os valores de umidade e pH obtidos na parte interna dos tratamentos ficaram dentro dos parâmetros recomendados para presunto curado. A cultura adicionada apresentou melhor desenvolvimento na parte interna da paleta. A temperatura de armazenamento recomendada para a paleta seria entre 5 e $10{ }^{\circ} \mathrm{C}$, uma vez que os valores de $\mathrm{pH}$ foram maiores que 5,2 e os de $\mathrm{a}_{\mathrm{w}}$ menores que 0,91 .

Palavras-chave: paleta suína; cultura pura; atividade de água; $\mathrm{pH}$; umidade.
\end{abstract}

\begin{abstract}
Ten boned and skinned pork butts weighing about $1.6 \mathrm{~kg}$ were injected with brine $\left(20 \% \mathrm{w} \cdot \mathrm{v}^{-1}\right)$ containing a pure culture of Staphylococcus xylosus, and were stored for 30 days in a chamber with controlled moisture ( 45 to 55\%) and temperature $\left(4\right.$ to $9{ }^{\circ} \mathrm{C}$ ). After this period, the butts were vacuum-packed and stored at $10^{\circ} \mathrm{C}$ for 120 days. Samples were taken in triplicate during processing and storage from the surface and internal parts of the cuts to test for water activity, $\mathrm{pH}$, moisture and plate counting of pure culture, in order to characterize and associate the processed product with other fermented meat products. The internal moisture and $\mathrm{pH}$ of the cured, matured and fermented pork butts during storage fell within the range recommended for cured ham. The culture added to the product showed a better development in the internal portion of the pork cuts. The recommended storage temperature for the product was found to be 5 to $10{ }^{\circ} \mathrm{C}$, since the pH values were higher than 5.2 and water activity lower than 0.91

Keywords: pork shoulder butt; pure culture; water activity; pH; moisture.
\end{abstract}

\section{Introdução}

Os produtos cárneos fermentados são caracterizados pela adição de culturas puras de microrganismos específicos que provocam alterações bioquímicas desejáveis ${ }^{9}$. O conhecimento dos fatores que influenciam no desenvolvimento e nas transformações provocadas por esses microrganismos auxilia na melhoria da qualidade e na obtenção de novos produtos cárneos fermentados. $\mathrm{O}$ conteúdo de água livre, umidade e $\mathrm{pH}$ influenciam nas modificações físicas e químicas e também na multiplicação dos microrganismos, influenciando assim na qualidade e estabilidade desses produtos ${ }^{14}$. Como conseqüência, o conhecimento dos valores de atividade de água $\left(\mathrm{a}_{\mathrm{w}}\right)$, umidade, e pH é fundamental, pois esses dados estão correlacionados

\footnotetext{
Recebido para publicação em 30/11/2006

Aceito para publicação em 24/7/2007 (002105)

Departamento de Ciência e Tecnologia de Alimentos,

Centro de Ciências Rurais - CCR,

Universidade Federal de Santa Maria - UFSM, Santa Maria - RS, Brasil

Curso de Pós-graduação em Tecnologia de Alimentos,

Universidade Federal do Paraná - UFPR, Centro Politécnico,

Jardim das Américas, Usina Piloto, Bloco A, Sala PO17, CP 19011

CEP 81531-990, Curitiba - PR, Brasil

${ }^{3}$ Curso de Engenharia de Alimentos, Universidade Regional Integrada - URI

Av. Sete de setembro, 1621, CEP 99700-000, Erechim - RS, Brasil,

E-mail:ajc@uricer.edu.br

*A quem a correspondência deve ser enviada
}

com o desenvolvimento das culturas adicionadas e com as suas atividades metabólicas ${ }^{7}$.

Durante a etapa de secagem e maturação do presunto curado, considerado um produto fermentado, $\mathrm{a}_{\mathrm{w}}$ diminui possibilitando assim a conservação do produto. Esta etapa desempenha importante papel no desenvolvimento de algumas características como textura, cor e sabor, pois ocorrem várias reações químicas e modificações em sua estrutura ${ }^{12}$. Essa redução do teor de umidade implica numa diminuição automática no valor de $\mathrm{a}_{\mathrm{w}}$, impedindo o desenvolvimento de outros microrganismos deteriorantes e a conseqüente alteração de suas características ${ }^{2}$.

A maioria dos microrganismos, incluindo as bactérias patogênicas, se desenvolve rapidamente a níveis de $\mathrm{a}_{\mathrm{w}}$ entre 0,99 a 0,986. Espécies da família Enterobacteriaceae, como o Proteus vulgaris e Serratia liquefaciens, quando se desenvolvem provocam alterações em presuntos curados, facilmente visualizadas como produção de gás em porções internas. Proteus vulgaris apresenta como exigências mínimas para multiplicação a temperatura de $15{ }^{\circ} \mathrm{C}$ e valor de $\mathrm{a}_{\mathrm{w}}$ de 0,95 , enquanto que a $10{ }^{\circ} \mathrm{C}$ o valor de $\mathrm{a}_{\mathrm{w}}$ necessário é pelo menos 0,93 . Serratia liquefaciens em temperatura de $8{ }^{\circ} \mathrm{C}$ necessita de $\mathrm{a}_{\mathrm{w}}$ mínima de 0,94 para conseguir se desenvolver, e a $6{ }^{\circ} \mathrm{C}$ o valor diminui para $0,93^{3}$. Em presuntos curados foram en- 
contrados valores de $\mathrm{a}_{\mathrm{w}} 0,88$ e 0,89 na parte interna ${ }^{17}$, e entre 0,80 a 0,84 em superfície ${ }^{11}$.

Staphylococcus coagulase negativa ( $\mathrm{SCN}$, como S. equorum, S. xylosus, S. saprophyticus, S. simulans, S. capitis, entre outros) são microrganismos pertencentes à família Micrococcaceae, e desejáveis em produtos cárneos fermentados por estarem envolvidos no desenvolvimento de características organolépticas específicas como sabor, aroma, textura e cor ${ }^{8}$. Em "Jerked Beef" com 16,5\% de sal e $a_{w}$ 0,74, SCN, corresponderam a $86 \%$ do total dos microrganismos presentes ${ }^{13}$. Em outro produto cárneo fermentado, denominado Cecina (elaborado com carne bovina e de origem espanhola), com $13,3 \%$ de sal e $\mathrm{a}_{\mathrm{w}} 0,88$, SCN representaram $81 \%$ da microbiota $^{8}$. A temperatura recomendada para o armazenamento de produtos cárneos é determinada levando-se em consideração os valores de $\mathrm{pH}$ e $\mathrm{a}_{\mathrm{w}}$ apresentados. Considerando isso, esses produtos podem ser divididos em três grupos: a) muito perecível; com $\mathrm{pH}>5,2 \mathrm{e} \mathrm{a}_{\mathrm{w}}>0,95$, sendo a temperatura de armazenamento recomendada $\leq 5{ }^{\circ} \mathrm{C}$; b) perecível; com $\mathrm{pH}$ entre 5,2 até 5,0 e $\mathrm{a}_{\mathrm{w}}$ entre 0,95 e até 0,90 , com temperatura recomendada $\leq 10{ }^{\circ} \mathrm{C}$; e c) estáveis; com $\mathrm{pH} \leq 5,2$ e $\mathrm{a}_{\mathrm{w}} \leq 0,95$ ou somente $\mathrm{pH}<5,0$ ou $\mathrm{a}_{\mathrm{w}} 0,91$, não sendo recomendada refrigeração para conservação ${ }^{6,15}$. GARCÍA et al. ${ }^{8}$ enfatizam que os produtos que podem ser armazenados à temperatura ambiente são os que apresentam valor de $\mathrm{a}_{\mathrm{w}} 0,86$, por possuírem excelente estabilidade microbiológica.

Acompanhou-se nesse trabalho a evolução dos valores de $\mathrm{a}_{\mathrm{w}}$, umidade, e da cultura de $S$. xylosus adicionada, durante a fase de processamento e armazenamento, e $\mathrm{pH}$ somente durante o armazenamento, da paleta suína curada maturada e fermentada, na superfície e na porção interna, tendo como objetivo a caracterização e as possíveis associações.

\section{Material e métodos}

\subsection{Tratamentos}

Dez peças de paleta suína sem pele e sem camada de gordura, apresentando em média peso de 1,6 $( \pm 0,2) \mathrm{kg}$, largura de $20( \pm 5) \mathrm{cm}$, comprimento de $25( \pm 6) \mathrm{cm}$ e espessura de $8( \pm 2) \mathrm{cm}$, foram selecionadas para a realização dos tratamentos. Uma solução primária de salmoura foi preparada 18 horas antes da utilização, constituída de $25 \%$ de $\mathrm{NaCl}$ (Cisne Extra Iodado), 1,25\% de sais de cura (cura 101, Duas Rodas), $1,12 \%$ de eritorbato de sódio (fixador de cor 302, Duas Rodas), $72,63 \%$ de água, e armazenada a $5{ }^{\circ} \mathrm{C}$. Uma cultura de S. xylosus (Linhagem Flora Carn SX ${ }^{\circledR} 100$, Chr. Hansen Ind. e Com) foi hidratada em água não clorada, na proporção de $4,5 \mathrm{~g} \mathrm{em} 36 \mathrm{~mL}$ de água, à temperatura de $15{ }^{\circ} \mathrm{C}$ durante 30 minutos, e adicionada à salmoura previamente preparada. Em seguida, a salmoura adicionada da cultura foi injetada nas peças de paleta na proporção de $20 \%$ (v/p), e as peças colocadas no "Tambler" a vácuo, onde permaneceram durante uma hora (30 minutos em movimento a $32 \mathrm{rpm}$ e 30 minutos paradas). Decorrido o tempo necessário, as peças foram levadas à câmara de maturação permanecendo durante trinta dias, com variação de temperatura entre 4 e $9{ }^{\circ} \mathrm{C}$, e de umidade relativa de 55 a $45 \%$. Passados os trinta dias dentro da câmara, as paletas fo- ram embaladas a vácuo em sacos plásticos coextrudado-nylon poli-cinco camadas com espessura de $100 \mu$ e armazenadas a $10{ }^{\circ} \mathrm{C}( \pm 2)$ durante 120 dias. As temperaturas adotadas no processamento e armazenamento tiveram como base o estudo efetuado sobre jamón curado ${ }^{2,4,6,10,11,12,17,18}$.

Durante as etapas de processamento e armazenamento, amostras das paletas foram coletadas para determinação dos valores de $\mathrm{a}_{\mathrm{w}}, \mathrm{pH}$, umidade e contagem de $\mathrm{S}$. xylosus. Em cada coleta, uma paleta foi selecionada, da qual foram retiradas

Tabela 1. Fluxo de produção, pontos das coletas (PC), dias das coletas e corridos, temperatura e umidade relativa de trabalho.

\begin{tabular}{|c|c|}
\hline $\begin{array}{l}\text { Pontos } \\
\text { de } \\
\text { coletas } \\
\text { (PC) }\end{array}$ & $\begin{array}{c}\text { Dias de coletas corridos, durante a fase de processa- } \\
\text { mento e armazenamento, temperatura e umidade relativa } \\
\text { de trabalho }\end{array}$ \\
\hline & Etapa de processamento \\
\hline $\mathrm{PC} 1$ & $5^{\circ} \mathrm{C}$ (matéria-prima) \\
\hline $\mathrm{PC} 2$ & $5{ }^{\circ} \mathrm{C}$ (após injeção e tambleamento) \\
\hline PC3 & $4{ }^{\circ} \mathrm{C}( \pm 2,0)$ e $55 \%( \pm 6,0)$ (após 2 dias dentro da câmara) \\
\hline PC4 & $4{ }^{\circ} \mathrm{C}( \pm 2,0)$ e $55 \%( \pm 6,0)$ (após 10 dias dentro da câmara) \\
\hline PC5 & $6{ }^{\circ} \mathrm{C}( \pm 1,0)$ e $47 \%( \pm 2,0)$ (após 20 dias dentro da câmara) \\
\hline \multirow[t]{2}{*}{ PC6 } & $9{ }^{\circ} \mathrm{C}( \pm 1,0)$ e $45 \%( \pm 4,0)$ (após 30 dias dentro da câmara) \\
\hline & Etapa de armazenamento \\
\hline PC7 & $\begin{array}{l}10^{\circ} \mathrm{C}( \pm 2,0) \text { (após } 30 \text { dias de armazenamento e } 60 \text { dias } \\
\text { corridos) }\end{array}$ \\
\hline PC8 & $\begin{array}{l}10^{\circ} \mathrm{C}( \pm 2,0) \text { (após } 60 \text { dias de armazenamento e } 90 \text { dias } \\
\text { corridos) }\end{array}$ \\
\hline PC9 & $\begin{array}{l}10^{\circ} \mathrm{C}( \pm 2,0) \text { (após } 90 \text { dias de armazenamento e } 120 \text { dias } \\
\text { corridos) }\end{array}$ \\
\hline $\mathrm{PC} 10$ & $\begin{array}{l}10^{\circ} \mathrm{C}( \pm 2,0) \text { (após } 120 \text { dias de armazenamento e } 60 \text { dias } \\
\text { corridos) }\end{array}$ \\
\hline
\end{tabular}

3 amostras superficiais (espessura de $2 \mathrm{~mm}$ ) e 3 da parte interna (no centro das peças). O fluxo de produção e os pontos de coleta na fase de processamento e de armazenamento, os dias corridos e os dias de coleta, a temperatura e a umidade relativa de trabalho são apresentados na Tabela 1.

\subsection{Análises físico-químicas}

\section{Determinação da atividade de água $\left(a_{w}\right)$}

A atividade de água de cada amostra foi determinada à temperatura de $30{ }^{\circ} \mathrm{C}$, utilizando-se o aparelho Aqua-lab, modelo CX-2.

\section{Determinação da umidade e $\mathrm{pH}$}

A umidade e o $\mathrm{pH}$ foram determinados conforme metodologia descrita pela $\mathrm{AOAC}^{1}$. Embora o $\mathrm{pH}$ tenha sido determinado nos dez pontos de coleta (PC), nesse trabalho serão apresentados somente os valores de PC6, PC7, PC8, PC9 e PC10.

\subsection{Análise microbiológica}

\section{Contagem de Staphylococcus xylosus}

As amostras obtidas foram diluídas em água peptonada e semeadas em duplicata em pour plate em agar mannitol salt 
(MSA), com incubação a $30{ }^{\circ} \mathrm{C}$ por 72 horas ${ }^{16}$. Em seguida, as colônias formadas foram enumeradas e os resultados finais foram convertidos em $\log _{10}$ e expressos em log de Unidade Formadora de Colônias.gr ${ }^{-1}\left(\log \mathrm{UFC}_{\text {.gr }}{ }^{-1}\right)$.

\subsection{Análise estatística}

Os resultados obtidos em cada ponto de coleta, na superfície e na parte interna da paleta, foram analisados estatisticamente por análise de variância e teste de Tukey com nível de significância de $5 \%$ para verificação de diferenças significativas $(\mathrm{p}<0,05)^{5}$.

\section{Resultados e discussão}

A Tabela 2 apresenta a evolução dos teores de umidade na superfície e na parte interna das peças de paleta analisadas durante as etapas de processamento e armazenamento. Na superfície da paleta, os teores de umidade variaram entre 75,5 e $39,9 \%$ durante a etapa de processamento (PC1 a PC6) e entre 37,5 e 46,7\% durante o armazenamento (PC7 a PC10), enquanto que na parte interna esses valores variaram entre 75,5 e $69,5 \%$ durante o processamento e entre 52,1 e $59,8 \%$ durante o armazenamento. A partir do segundo dia o teor de umidade na superfície da paleta passou a ser significantemente menor em relação ao da parte interna (Tabela 2).

Após o processamento, presuntos curados chineses apresentaram valores de umidade entre 40 e $50 \%^{18}$. O valor de umidade encontrado na superfície da paleta, após processamento (PC6), foi menor do que o valor mínimo encontrado no presunto curado chinês, mas após 90 e 120 dias de armazenamento os valores ficaram dentro do intervalo encontrado para esse produto (Tabela 2). Esse aumento de umidade ocorrido na superfície foi provocado pelo emprego da embalagem a vácuo, que proporciona um ambiente praticamente fechado e não permite a perda de umidade para o exterior, ocorrendo migração de água da parte mais concentrada para a menos concentrada ${ }^{2}$.

Conteúdo de umidade entre 57 e $67 \%$, na parte interna dos presuntos curados espanhóis, é considerado alto ${ }^{4}$. Depois de processada (PC6), a paleta apresentou teor de umidade na parte interna superior a 67\%, mas durante o armazenamento, após 60 dias (PC8), os valores passaram a diminuir atingindo no $120^{\circ}$ dia (PC10) o valor de $57,1 \%$ (Tabela 2 ). Para reduzir ainda mais o valor de umidade na parte interna da paleta, poderiam ser empregados vários procedimentos, mas nesse tipo de processamento empregado o melhor seria o aumento do período de processamento para 40 dias.

Os valores de $\mathrm{a}_{\mathrm{w}}$ e das contagens de $\mathrm{S}$. xylosus na superfície e na parte interna das peças de paleta estão na Figura 1.

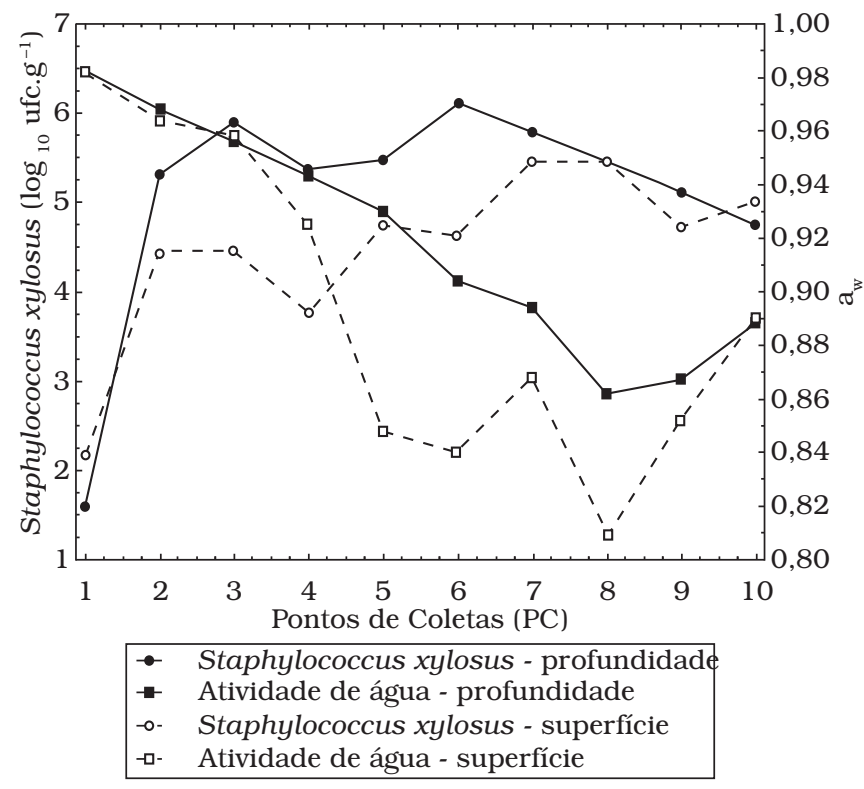

Figura 1. Valores médios de contagens de Staphylococcus xylosus e de atividade de água $\left(\mathrm{a}_{\mathrm{w}}\right)$ obtidos em superfície e na parte interna de peças de paleta suína durante as etapas de processamento (PC1 a PC6) e armazenamento (PC7 a PC10).

Os valores de $\mathrm{a}_{\mathrm{w}}$ na superfície das peças de paleta variaram entre 0,98 e 0,80, e na parte interna entre 0,98 e 0,86 (Figura 1). A partir do PC2 os valores de $\mathrm{a}_{\mathrm{w}}$ encontrados em superfície e na parte interna das peças de paleta foram inferiores ao da faixa considerada ideal para o desenvolvimento da maioria dos microrganismos patogênicos, que varia entre 0,99 e 0,986. Valores de $\mathrm{a}_{\mathrm{w}}$ inferiores a 0,93 são considerados inadequados para o desenvolvimento de grande parte de espécies da família Enterobacteriaceae, grupo freqüentemente utilizado como indicador higiênico de presuntos curados ${ }^{10}$. Os valores de $\mathrm{a}_{\mathrm{w}}$ encontrados na paleta a partir de PC4 na superfície e PC6 (parte interna) foram inferiores a esse valor, demonstrando que essa característica apresentada pelos tratamentos funcionaria como um obstáculo frente a essas bactérias (Figura 1).

Tabela 2. Valores médios ( \pm desvio padrão) dos teores de umidade das 3 repetições de amostras de superfície e interior de paletas suínas maturadas durante as etapas de processamento e armazenamento.

\begin{tabular}{|c|c|c|c|c|c|c|c|c|c|c|}
\hline & \multicolumn{10}{|c|}{ Pontos de coleta } \\
\hline & \multicolumn{6}{|c|}{ Processamento } & \multicolumn{4}{|c|}{ Armazenamento } \\
\hline & $\mathrm{PC} 1$ & PC2 & PC3 & PC4 & PC5 & PC6 & PC7 & PC8 & PC9 & PC10 \\
\hline & Dia 00 & Dia 00 & Dia 02 & Dia 10 & Dia 20 & Dia 30 & Dia 60 & Dia 90 & Dia 120 & Dia 150 \\
\hline Superfície & $\begin{array}{c}75,5^{\mathrm{a}} \\
( \pm 0,08)\end{array}$ & $\begin{array}{c}73,3^{\mathrm{a}} \\
( \pm 0,10)\end{array}$ & $\begin{array}{c}66,2^{\mathrm{b}} \\
( \pm 0,32)\end{array}$ & $\begin{array}{c}51,8^{\mathrm{b}} \\
( \pm 1,41)\end{array}$ & $\begin{array}{c}38,6^{\mathrm{b}} \\
( \pm 0,65)\end{array}$ & $\begin{array}{c}39,6^{\mathrm{b}} \\
( \pm 0,58)\end{array}$ & $\begin{array}{c}37,5^{\mathrm{b}} \\
( \pm 1,96)\end{array}$ & $\begin{array}{c}38,9^{\mathrm{b}} \\
( \pm 1,51)\end{array}$ & $\begin{array}{c}44,4^{\mathrm{b}} \\
( \pm 3,67)\end{array}$ & $\begin{array}{c}46,7^{\mathrm{b}} \\
( \pm 0,55)\end{array}$ \\
\hline Interior & $\begin{array}{c}75,5^{\mathrm{a}} \\
( \pm 0,08)\end{array}$ & $\begin{array}{c}74,2^{\mathrm{a}} \\
( \pm 1,97)\end{array}$ & $\begin{array}{c}77,3^{\mathrm{a}} \\
( \pm 0,84)\end{array}$ & $\begin{array}{c}73,4^{\mathrm{a}} \\
( \pm 0,10)\end{array}$ & $\begin{array}{c}71,6^{\mathrm{a}} \\
( \pm 0,11)\end{array}$ & $\begin{array}{c}69,5^{\mathrm{a}} \\
( \pm 0,36)\end{array}$ & $\begin{array}{c}59,8^{\mathrm{a}} \\
( \pm 0,96)\end{array}$ & $\begin{array}{c}52,1^{\mathrm{a}} \\
( \pm 0,73)\end{array}$ & $\begin{array}{c}56,4^{\mathrm{a}} \\
( \pm 1,15)\end{array}$ & $\begin{array}{c}57,1^{\text {a }} \\
( \pm 1,29)\end{array}$ \\
\hline
\end{tabular}

Letras diferentes na mesma coluna indicam diferença significativa $(p<0,05)$. 
Na fase de processamento (PC1 a PC6), as contagens de S. xylosus variaram entre 2,17 log UFC.gr ${ }^{-1}$ e 4,73 log UFC.gr ${ }^{-1}$ (superfície) e entre 1,60 log UFC.gr ${ }^{-1}$ e 6,11 log UFC.gr ${ }^{-1}$ (parte interna). Durante o armazenamento (PC7 a PC10), a variação foi de 4,62 log UFC.gr ${ }^{-1}$ a 5,47 log UFC.gr ${ }^{-1}$ (superfície) e 4,74 log UFC.gr-1 a 5,78 log UFC.gr-1 (parte interna) (Figura 1).

Entre PC6 e PC10, os valores de $\mathrm{a}_{\mathrm{w}}$ na superfície dos tratamentos variaram entre 0,80 e 0,89 , enquanto na parte interna variaram entre 0,86 e 0,90 . Mesmo sendo considerados baixos esses valores de $\mathrm{a}_{\mathrm{w}}$ para o desenvolvimento de bactérias cresceram colônias do Staphylococcus xylosus na superfície e na parte interna da paleta suína curada maturada e fermentada (Figura 1), fato semelhante ocorreu com GARCÍA et al. ${ }^{8} \mathrm{e}$ $\mathrm{PINTO}^{13}$, que também observaram desenvolvimento de SCN em baixos valores de $\mathrm{a}_{\mathrm{w}}$, como 0,88 e 0,74, respectivamente.

Caracterizada a paleta suína curada, maturada e fermentada em relação aos valores de $\mathrm{a}_{\mathrm{w}}$ e pH, elaborou-se a Figura 2 com o objetivo de associar esses valores à classificação citada por CARRASCOSA et al. ${ }^{6}$ e SABATAKOU et al. ${ }^{15}$, que determinam a temperatura de armazenamento baseados em valores de $a_{w}$ e $p H$. A paleta suína curada, maturada e fermentada poderia ser armazenada em temperatura ambiente levandose em conta que os valores de $\mathrm{a}_{\mathrm{w}}$ encontrados em superfície e na parte interna no final do processamento (PC6) e durante o armazenamento (PC7 a PC10) foram menores que 0,91. Porém, caso fossem considerados somente os valores de $\mathrm{pH}$ nas mesmas fases de produção e armazenamento, a paleta suína não deveria ser armazenada à temperatura ambiente, e sim abaixo de ou a $5{ }^{\circ} \mathrm{C}$, por apresentar valores superiores a 5,2 (Figura 2). GARCÍA et al. ${ }^{8}$ mencionam que para que os produtos cárneos

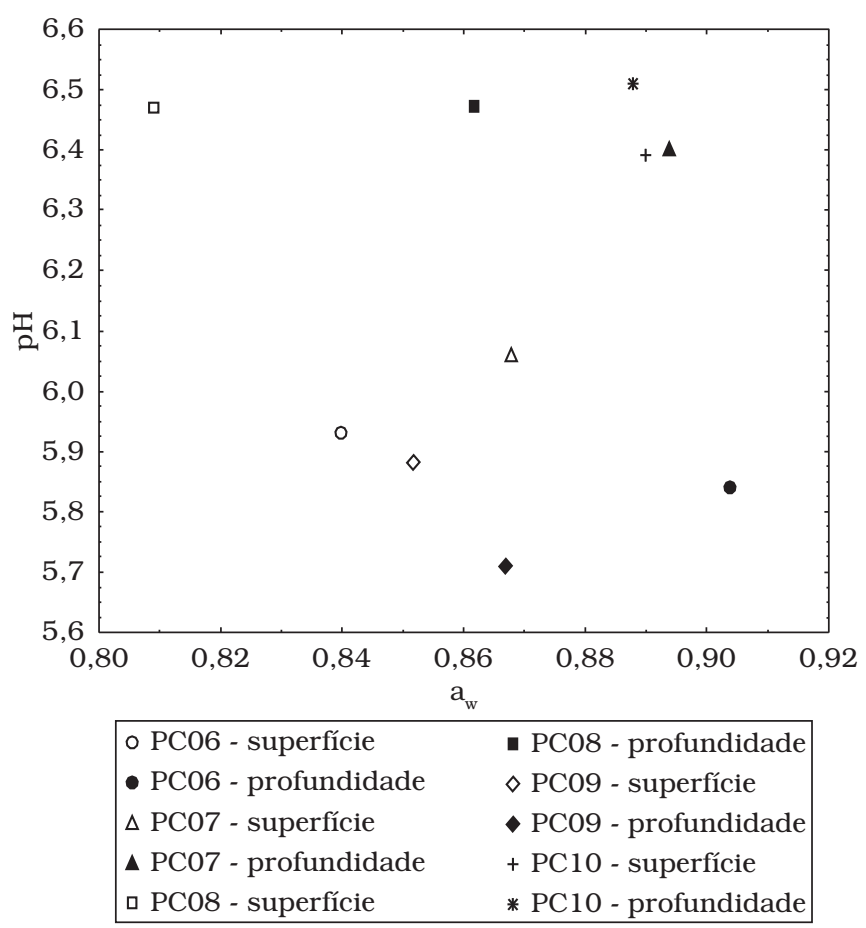

Figura 2. Valores de atividade de água e $\mathrm{pH}$ na superfície e na parte interna da paleta suína, no produto final (PC6) e durante o armazenamento (PC7 a PC10). sejam armazenados à temperatura ambiente é necessário que os mesmos apresentem valor de $\mathrm{a}_{\mathrm{w}} 0,86$. Associando esse parâmetro aos valores de $\mathrm{pH}$ apresentados pela paleta suína durante o período de armazenamento (Figura 2), pode-se concluir que a temperatura de armazenamento da paleta seria entre 5 e $10{ }^{\circ} \mathrm{C}$, uma vez que os valores de $\mathrm{pH}$ foram maiores que 5,2 e os de $\mathrm{a}_{\mathrm{w}}$ menores do que 0,91 .

Agora vamos associar os valores de $\mathrm{pH}$ apresentados na Figura 2 com a cultura empregada na elaboração da paleta.

Staphylococcus xylosus caracteriza-se por ser aeróbico, podendo também ser anaeróbico facultativo, com bom desenvolvimento em superfície dos salames durante a fase de processamento, devido aos altos valores de $\mathrm{pH}$ decorrentes do crescimento dos $\operatorname{mofos}^{9}$. Na paleta suína curada, maturada e fermentada no produto final (PC6) e durante a fase de armazenamento (embalagem a vácuo), ocorreu maior desenvolvimento de S. xylosus na parte interna, onde os valores de $\mathrm{pH}$ foram semelhantes ao da superfície (Figura 2), além de maiores valores de $\mathrm{a}_{\mathrm{w}}$ e umidade (Tabela 2).

$\mathrm{O}$ valor de $\mathrm{pH}$ na parte interna da paleta, no final do processamento (PC6) foi 5,84, e durante o armazenamento variou entre 6,40 e 6,51 (PC7 a PC10) (Figura 2). Em presuntos curados considerados adequados ao consumo, no final do processamento foram encontrados valores de $\mathrm{pH}$ entre 5,6 e $6,4^{2}$, sendo que apenas no $120^{\circ}$ dia de armazenamento (PC10) foram observados valores de $\mathrm{pH}$ acima desse limite (Figura 2). $\mathrm{O}$ valor de $\mathrm{pH}$ apresentado no $120^{\circ}$ dia de armazenamento $(6,51)$, indicaria que não seria aconselhável o consumo da paleta suína, uma vez que esse valor indicaria uma possível deterioração ${ }^{2,6}$. A elevação de $\mathrm{pH}$ observada durante a etapa de armazenamento estaria principalmente relacionada à ação de enzimas proteolíticas endógenas e de origem bacteriana (S. xylosus).

\section{Conclusões}

Considerando os resultados obtidos, observou-se que os valores de umidade na parte interna da paleta suína curada, maturada e fermentada durante a etapa de armazenamento, apresentaram-se dentro dos parâmetros recomendados para os presuntos curados espanhóis, assim como os valores de pH obtidos após 60 dias de processamento e até o $90^{\circ}$ dia de armazenamento. Mesmo com baixa $\mathrm{a}_{\mathrm{w}}$, a cultura de $S$. xylosus conseguiu se desenvolver tanto na superfície como na parte interna dos tratamentos, e os valores de $\mathrm{pH}$ obtidos na parte interna da paleta suína curada, maturada e fermentada, permitem a recomendação de armazenamento do produto entre 5 e $10{ }^{\circ} \mathrm{C}$.

\section{Referências bibliográficas}

1. AOAC. Association Official Analytical Chemists. Official methods of analysis. $17^{\text {th }}$ edition, $4^{\text {th }}$ revision. Maryland, USA, 2002.

2. ARNAU, J.; HUGAS, M.; MONFORT, J. M. El jamón curado: Aspectos técnicos Girona, Itália, Grafis-Sant S. A. 1987, 352 p.

3. BARBUTI, S.; PAROLARI, G. Causes and prevention of dry-cured ham defects. In: II Symposium Internacional del Jamón Curado. Anais... Barcelona, Espanha, março, 2000. p. 19-25. 
4. BLANCO, D. et al. Physico-chemical characterization of "bone Taint" in Spanish dry-cured hams. Journal of Food Protection. v. 60, n. 6, p. 667-672, 1997.

5. CAMPOS, G. H. Estatística experimental não paramétrica. 4. ed. Piracicaba, São Paulo: ESALQ, 1983. 349 p.

6. CARRASCOSA, A. V.; CORNEJO, I. Aspectos físico-químicos del curado de jamón serrano y su influencia sobre el desarrollo microbiano (Revisión). Aliementaria, p. 27-33, 1989.

7. CHIRIFE, J.; BUERA, M. P. Water Activity, Water Glass Dynamics, and the Control of Microbiological Growth in Foods. Critical Reviews in Food Science and Nutrition, v. 36, n. 5, p. 465-513, 1996.

8. GARCÍA, I.; ZUMALACÁRREGUI, J. M.; DÍEZ, V. Microbial succession and identification of Micrococcaceae in dried beef cecina, an intermediate moisture meat product. Food Microbiology v. 12, n. 1, p. 309-315, 1995

9. LÜCKE, F. K. Fermented meat products. Food Research International. v. 27, n. 3, p. 299-307, 1994.

10. MARÍN, M. E.; CARRASCOSA, A. V.; CORNEJO, I. Characterization of Enterobacteriaceae strains isolated during industrial processing of dry-cured hams. Food Microbiology, v. 13, n. 5, p. 375-381, 1996.

11. MARTín, A. et al. Contribución de la población microbiana a la seguridad y calidad del jamón curado. In: II Symposium Internacional del Jamón Curado. Anais... Barcelona, Espanha, marzo, 2000. p. 55-64

12. PÉREZ-ALVAREZ, J. A. et al. Spanish Dry-Cured Ham Aging Process: Colour Characteristics. In: INTERNATIONAL
CONGRESS OF MEAT SCIENCE AND TECHNOLOGY, 44, 1998. Espanha. Proceedings... Dry-cured products, Barcelona, 1998, C-80, p. 984-985.

13. PINTO, M. F. Culturas iniciadoras - Starters - no processamento de jerked beef, um derivado do charque. São Paulo, 1996, 93 p. Tese (Doutorado) - Faculdade de Ciências Farmacêuticas - Universidade de São Paulo.

14. ROCKLAND, L. B.; BEUCHAT, L. R. Water Activity: Theory and Applications to Food. Marcel Dekker, New York, Inc. 1987, 404 p.

15. SABATAKOU, O. et al. Classification of Greek meat products on the basis of $\mathrm{pH}$ and $\mathrm{A}_{\mathrm{w}}$ values. Fleischwirtschaft, v. 18, n. 8, p. $91-95,2001$.

16. TAHNKE, L. H. Dried Sausages fermented with Staphylococcus xylosus at different temperatures and with different ingredient levels - Part I. Chemical and bacteriological data. Meat Science, v. 41, n. 2, p. 179-191, 1995.

17. VANDENDRIESSCHE F. I. R. The Northern and Southern European type of dry-cured hams in the Belgian market. In: El jamón curado: Tecnología y análisis de consumo. Simpósio Especial - 44th ICoMST. Anais..., Barcelona, Espanha, 1998. p. $148-151$.

18. ZHU, S. Dry-cured ham in China. El jamón curado: Tecnología y análisis de consumo. In: El jamón curado: Tecnología y análisis de consumo. Simpósio Especial - 44th ICoMST. Anais..., Barcelona, Espanha, 1998. p. 185-188. 